\title{
The Kyphosis-lordosis Difference Parameter and Its Utility in Understanding the Pathogenesis of Adolescent Idiopathic Scoliosis
}

\section{Adrian Gardner ( $\square$ adrian.gardner@nhs.net)}

Royal Orthopaedic Hospital NHS Foundation Trust https://orcid.org/0000-0001-6532-7950

Fiona Berryman

Royal Orthopaedic Hospital NHS Trust: Royal Orthopaedic Hospital NHS Foundation Trust

Paul Pynsent

University of Birmingham Edgbaston campus: University of Birmingham

\section{Research Article}

Keywords: Kyphosis, Lordosis, Scoliosis, AIS, Sagittal, Spine

Posted Date: February 23rd, 2022

DOI: https://doi.org/10.21203/rs.3.rs-1155667/v1

License: (1) This work is licensed under a Creative Commons Attribution 4.0 International License.

Read Full License 


\section{Abstract \\ Objective}

The relationship of sagittal spinal shape in the pathogenesis of adolescent idiopathic scoliosis (AIS) is recognised. What is not clear is the relationship between the sagittal shape of those without scoliosis and the potential development of AIS, including the greater prevalence in females. The use of a new parameter, the kyphosis-lordosis $(\mathrm{KL})$ difference, was developed to explore this further.

\section{Results}

The KL difference was calculated for 117 males and 79 females over seven years with 831 measures made. For females, the $\mathrm{KL}$ difference, between the ages of 9 and $12 \frac{1}{2}$ years, decreases from $5^{\circ}$ to nearly $0^{\circ}$ until starting to climb again from the age of 14 years, back to $5^{\circ}$ by the age of $16 \frac{1}{2}$ years. For males, there is a gradual decline from $9^{\circ}$ at age 9 years to $5^{\circ}$ at age 17 years. Both age and sex were statistically significant in the development of the parameter. When comparing to previously published data around the true, de-rotated, sagittal shape of the scoliotic spine, the KL difference has utility in explaining the female predominance in the prevalence of AIS. This adds to the weight of evidence behind understanding why AIS develops.

\section{Introduction}

The development of the sagittal shape of the spine has been advanced as a hypothesis for the development of adolescent idiopathic scoliosis (AIS) [1, 2, 3]. However, information on the development of the sagittal shape of the spine in adolescents without scoliosis is less well described [4]. Linking the development of the shape of the spine in those with, and without, AIS is critical to the understanding of why some children develop AIS, and some do not.

Our group has previously described the development of kyphosis and lordosis in the developing spine in those without scoliosis in a longitudinal fashion over 5 years [5] using surface topography (Integrated Shape Imaging System 2- ISIS2 [6]). This showed that age was statistically significant for both kyphosis and lordosis and sex was only significant in the development of lordosis. A re-examination and reanalysis of kyphosis and lordosis modelling was performed to investigate the relationship of kyphosis and lordosis, when viewed together as one parameter. This paper shows the development of this new parameter with age, between males and females and explains the potential relevance to the pathogenesis of scoliosis.

\section{Background And Ethical Considerations}

This is a re-analysis of prospectively collected longitudinal data of spinal and back shape following two further years of data collection. Ethical approval was via the NRES committee West Midlands - South 
Birmingham (11/H1207/10). The investigation was of a group of children without spinal deformity who were imaged using the ISIS2 surface topography system over a seven year period. ISIS2 is a radiation free method of gaining 3D information of the shape of the back and spine. The development of kyphosis and lordosis of this group after 5 years of measures has previously been reported [5]. Measures of spinal shape from ISIS2 include measures of kyphosis and lordosis, measured using a technique analogous to the Cobb method [7]. Kyphosis is measured between the vertebra prominens (VP) and the point of inflection between the sagittal curves and lordosis is measured between the point of inflection and the sacrum. By definition, both kyphosis and lordosis are measured in degrees.

\section{Methods}

Using a larger data set, the modelling of the development of kyphosis and lordosis was re-examined using the methods previously described [5]. The kyphosis-lordosis (KL) difference was then calculated by subtracting the lordosis from the kyphosis of each individual. The measures of kyphosis, lordosis and KL difference were analysed longitudinally against age with linear mixed effect modelling [8], to allow for repeated measures from the same individual over time, using the Ime4 [8] and ggplot2 [9] packages in $\mathrm{R}$ [10]. Linear mixed effect models were produced to examine the effects of age and sex in the KL difference parameter. This was done by producing a model with the fixed effects of age and sex and the random effect of repeat measures. To examine either the effects of age or sex, a model without that parameter was also produced and compared using an ANOVA method to the model with both parameters [11]. Significance was predefined as $p<0.05$. Following the review of the Akaike information criterion [12] for potential model types, a linear analysis was chosen as more complicated non-linear models did not improve the behaviour of the model.

\section{Results}

The group analysed consisted of 117 males and 79 females. Over the seven year measurement period there were 831 measures made. Not all children were measured at every occasion, although 166 participants were measured on three or more occasions, comprising 787 of the total number of measures.

The age range of the participants for all of the measurements taken was between 9 and 17 years. When all of the measurements were analysed individually, the mean height was $1.57 \mathrm{~m}$ (SD $0.13 \mathrm{~m}$, range 1.30 to $1.88 \mathrm{~m}$ ) for males and $1.55 \mathrm{~m}$ (SD $0.12 \mathrm{~m}$, range 1.18 to $1.84 \mathrm{~m}$ ) for females and the body mass index (BMI) was $20.4 \mathrm{~kg} / \mathrm{m}^{2}$ (SD $3.2 \mathrm{~kg} / \mathrm{m}^{2}$, range 13.8 to $31.6 \mathrm{~kg} / \mathrm{m}^{2}$ ) for males and $19.7 \mathrm{~kg} / \mathrm{m}^{2}$ (SD 2.9 $\mathrm{kg} / \mathrm{m}^{2}$, range 14.0 to $33.1 \mathrm{~kg} / \mathrm{m}^{2}$ ) for females.

The participants were in the main of Caucasian descent with approximately $3 \%$ from a non-Caucasian heritage.

Figure 1 is a composite box and whisker plot of the $K L$ difference against age with Figure 2 showing the $\mathrm{KL}$ difference in the form of loess lines with the $95 \%$ confidence interval as a grey funnel around the loess 
line [13]. For females, the $\mathrm{KL}$ difference, between the ages of 9 and $12 \frac{1}{2}$ years, decreases from $5^{\circ}$ to nearly $0^{\circ}$ until starting to climb again from the age of 14 years back to $5^{\circ}$ by the age of $161 / 2$ years. For males, there is a gradual decline from $9^{\circ}$ at age 9 years to $5^{\circ}$ at age 17 years. A decreasing $\mathrm{KL}$ value indicates a relative increase in the size of the lordosis versus the kyphosis, or conversely, a relative decrease in kyphosis versus the value of the lordosis. The statistical analysis using the linear mixed effect modelling technique is shown in Table 1. For the $\mathrm{KL}$ difference, both age and sex were found to be statistically significant in the development of the parameter. The point of inflection was not found to have significant relationship with either kyphosis, lordosis or the $\mathrm{KL}$ difference.

Table 1

Statistical analysis of measured parameters against age and sex.

\begin{tabular}{|lll|}
\hline & Age & Sex \\
\hline Kyphosis & $<0.001$ & 0.0648 \\
\hline Lordosis & $<0.001$ & 0.009 \\
\hline Kyphosis Lordosis Difference & 0.016 & $<0.001$ \\
\hline
\end{tabular}

\section{Discussion And Conclusion}

There is a paucity in the understanding of the normal growth and development of children. Whilst growth standards have been published for parameters that can be measured repeatedly without risk to the child (such as the WHO standards for height and weight [14]), techniques that require the repeated use of ionising radiation have been avoided due to the cumulative risks to the subject [15]. As such, serial radiographic measures of spinal shape during growth in those without spinal deformity do not exist. Other measures, such as ISIS2 surface topography used in this, and previous works [5] (as a radiation free technique), allow an understanding of normal growth and development.

AIS is a condition where there is a 3D deformation of the shape of the spine, seen as an alteration of shape away from the norm in the coronal, sagittal and axial planes, with the development of scoliosis, a change of kyphosis and lordosis and the development of intervertebral rotation [16]. There is a growing literature that supports the hypothesis that AIS develops solely in humans and is not seen in other species because of the specific sagittal shape of the human spine $[2,3,17]$. This is thought to occur because of the inter-species difference in the shape of the pelvis with the associated development of the lumbar lordosis seen in humans [18].

AIS is more common in females than males [16]. The reasons for this are not clear. Previous research has suggested there may be a link to the differences in the development of the sagittal profile of the spine between the sexes [4]. Our previous work did not show a statistical difference in the effect of sex on the development of kyphosis, though visual inspection of the plot showed a difference in shape of the curves between males and females [5]. There was, however, a statistically significant difference with sex for the 
development of lumbar lordosis. However, linking the potential development the sagittal profile of the spine and the development of AIS [19] by considering only one segment of the spine in isolation would seem shortsighted. Previous literature in adult scoliosis [20] has noted that the relationships between kyphosis and lordosis in an individual with adult degenerative scoliosis is related to that individuals clinical outcome, but nothing similar performed in the AIS literature.

Consequently, the $\mathrm{KL}$ difference is a method that examines the magnitude of both the kyphosis and lordosis as a whole in an individual. The analysis presented here demonstrates that sex is highly statistically significant in the development of the $\mathrm{KL}$ difference. In females between the ages of 12 and 14 , the decrease seen shows that the mean value is only just positive with the $95 \%$ confidence interval crossing zero. As seen in Figure 2, there is a different shape to the plotted curves between males and females for the parameter.

It has recently become possible to create and manipulate a 3D model of the spine through computer processing and modelling of orthogonal radiographs from EOS imaging [21]. Using a local coordinate system for each vertebral body, the authors have been able to exclude axial rotation and lateral bend from 3D models of the scoliotic spine to reveal the true amount of kyphosis and lordosis. When the effects of axial rotation and lateral bend are removed, the 3D model has revealed that the true kyphosis is less than would be measured from a 2D radiograph of the same spine. When comparing the amount of kyphosis and lordosis from the 3D models with 3D models created from non-scoliotic spines, it was found that there is less kyphosis in those with scoliosis compared to the non-scoliotics (non-scoliotics mean of $55^{\circ}$ of kyphosis against a mean of $22^{\circ}$ for thoracic curves and $34^{\circ}$ for thoracolumbar curves) and more lordosis (non-scoliotics mean of $56^{\circ}$ of lordosis against a mean of $61^{\circ}$ for thoracic curves and $65^{\circ}$ for thoracolumbar curves). The $\mathrm{KL}$ difference parameter described here can be calculated using this data, with results of $-2^{\circ}$ for the non-scoliotics, $-39^{\circ}$ for thoracic curves and $-31^{\circ}$ for thoracolumbar curves, demonstrating the mismatch between kyphosis and lordosis in those with AIS. It is worth noting that the values of kyphosis and lordosis quoted by Newton [21] are higher than has been previously quoted in the literature [22, 23]. This is likely to reflect the differences in measurement between a 3D model of the entire kyphosis versus the 2D measure obtained from a radiograph, where the superior limit of measurement may not be the superior thoracic spine due to the difficulties of imaging through the shoulder girdle.

The lack of influence of the point of inflection in this analysis is as would be expected given the work reviewed by Sanders [23] where, after the age of 9 , the balance between the percentage contributions of the lengths of the thoracic spine and lumbar spine remain similar as age increases.

The results presented here add to the hypotheses described around the sagittal shape as an initiating factor for the development of AIS [1,2,3]. It is the totality of the sagittal spinal shape, rather than just the amount of kyphosis or lordosis that will contribute to the shape of the spine in the sagittal plane. This is because the curves of the kyphosis and lordosis must allow for an overall spinal balance that allows upright stance [24]. Consequently, the development with age of a situation where the relationship between the amount of kyphosis and lordosis changes will affect the overall sagittal shape, reflected by the 
changing value of the KL difference parameter. Given that the work of Newton [21] shows scoliosis to have a negative $\mathrm{KL}$ difference, and that the work presented here shows a difference between males and females with females having a smaller and, in some cases, a negative KL difference, there are similarities between the $\mathrm{KL}$ difference in a non-scoliotic and scoliotic population that may help to explain the sex distribution of AIS.

\section{Conclusion}

In conclusion, the work presented here describes how the KL difference, a new parameter that demonstrates that the overall sagittal shape of the thoracic and lumbar spines in children without spinal deformity, changes significantly with age and sex. This adds to the hypotheses around the development of the sagittal shape of the spine and the association to the development of AIS.

\section{Limitations}

The weakness of the ISIS2 technique is the inability to image individual vertebral bodies or to demonstrate the anatomical relationship of the pelvis to the spine and quantify any changes in pelvic rotation that may have occurred during the period of the study. To overcome this would require serial whole spine radiographs, including the pelvis, in growing children which causes a number of ethical issues such that it would never be performed. Also, this study did not analyse the effects of handedness, weight of school bags or a differing BMI on the development of the KL difference. Handedness has been found not to be significant previously in the development of kyphosis and lordosis separately [25] and thus is unlikely to be a factor in the $\mathrm{KL}$ difference. An increased BMI (>85 percentile for age) is known to increase kyphosis when compared to those with a normal BMI in AIS [26] so may have an effect on the $\mathrm{KL}$ difference in those without AIS. School bags do alter posture and spinal shape whilst carrying the weight [27]. It is not clear whether that effect is prolonged after the weight is removed and so no supposition can be made for the effect of school bags on the $\mathrm{KL}$ difference.

\section{List Of Abbreviations}

AIS

adolescence idiopathic scoliosis

$\mathrm{KL}$ difference

kyphosis-lordosis difference

ISIS2

Integrated Shape Imaging System 2

NRES

National Research Ethics Service UK

3D

3 dimensions

VP 
Vertebra prominens

ANOVA

Analysis of varience

SD

standard deviation

BMI

body mass index

WHO

World Health Organisation

2D

2 dimensions

\section{Declarations}

\section{Ethics approval and consent to participate}

Ethical approval was via the NRES committee West Midlands - South

Birmingham, UK (11H1207/10). Consent to participate was covered in that ethical approval.

\section{Consent for publication}

As the data is anonymised, consent for publication is not required.

\section{Availability of data and materials}

The data sets used and analysed during this study are available from the corresponding author on reasonable request.

\section{Competing interests}

The authors have no competing interests to declare, financial or non-financial.

\section{Funding}

There is no finding to declare.

\section{Authors' contributions}

AG conceived the study, analysed the data and wrote the first draft of the manuscript.

FB collected the data.

PP provided statistical oversight.

All authors read and approved the final manuscript. 
Acknowledgements

Not applicable.

\section{References}

1. Castelein R, van Dieën J, Smit T. The role of dorsal shear forces in the pathogenesis of adolescent idiopathic scoliosis - a hypothesis. Med Hypotheses. 2005;65:501-8.

2. Janssen M, Kouwenhoven J, Castelein R. The role of posteriorly directed shear loads acting on a prerotated growing spine: a hypothesis on the pathogenesis of idiopathic scoliosis. Stud Health Technol Inform. 2010;158:112-7.

3. Kouwenhoven J, Smit T, van der Veen A, et al. Effects of dorsal versus ventral shear loads on the rotational stability of the thoracic spine: a biomechanics porcine and human cadaveric study. Spine. 2007;32:2545-50.

4. Schlösser T, Vincken $K$, Rogers $K$, et al. Natural sagittal spino-pelvic alignment in boys and girls before, at and after the adolescent growth spurt. Eur Spine J. 2015;24:1158-67.

5. Gardner A, Berryman F, Pynsent P. The development of kyphosis and lordosis in the growing spine. Spine. 2018;43:E1109-15.

6. Berryman F, Pynsent P, Fairbank J, Disney S. A new system for measuring three-dimensional back shape in scoliosis. Eur Spine J. 2008;17:663-72.

7. Cobb J. Outline for the study of scoliosis. AAOS Instr Course Lect. 1948;5:261-75.

8. Bates D, Mächler M, Bolker B, Walker S. Fitting linear mixed-effects models using Ime4. J Stat Softw. 2015;67:1-48.

9. Wickham H. Elegant graphics for data analysis. New York: Springer Verlag; 2016.

10. R Core Team. R: A language and environment for statistical computing. R Foundation for Statistical Computing, Vienna, Austria. [Online]. Available: https://www.R-project.org/ (2020).

11. Winter B. Linear models and linear mixed effects models in $\mathrm{R}$ with linguistic applications. arXiv:1308.5499. [Online]. Available: http://arxiv.org/pdf/1308.5499.pdf. (2013). Accessed 10 Nov 2021.

12. Akaike H. A new look at the statistical model identification. IEEE Trans Automat Contr. 1974;19:71623.

13. Cleveland W, Grosse E, Shyu W. Local regression models. In: Chambers J, Hastie T, editors. Statistical Models in S. Wadsworth \& Brooks/Cole; 1992. pp. 309-76.

14. de Onis $\mathrm{M}$, Onyango A, Borghi $\mathrm{E}$, et al. Development of a WHO growth reference for school-aged children and adolescents. Bulletin World Health Organ. 2007;85:660-7.

15. Law M, Ma W-K, Lau D, et al. Cumulative radiation exposure and associated cancer risk estimates for scoliosis patients: impact of repetitive full spine radiography. Eur J Radiol. 2016;85:625-8. 
16. Nelson S, Sanders J. Idiopathic Scoliosis. In the SRS etext. [cited 10/5/2019]. Available from http://etext.srs.org/book/.

17. Sarwak J, Castelein R, Maqsood A, Aubin C. The biomechanics of induction in adolescent idiopathic scoliosis: theoretical factors. J Bone Joint Surg Am. 2019;e22:18-23.

18. Vital J, Sénégas J, Castelian J-E. From the head to the feet: anatomy of the upright position. In: Roussouly P, Pinheiro-Franco J, Labelle H, Gehrchen M, editors. Sagittal balance of the spine: From normal to pathology: A key for treatment strategy. New York:Thieme; 2019. p23-42.

19. Schlösser T, Shah S, Reichard S, et al. Differences in early sagittal plane alignment between thoracic and lumbar adolescent idiopathic scoliosis. Spine J. 2014;14:282-90.

20. Zhou S, Li W, Su T, et al. Does lumbar lordosis minus thoracic kyphosis predict the clinical outcome of patients with adult degenerative scoliosis? J Orthop Surg Res. 2019;14:290.

21. Newton P, Osborn E, Bastrom T, et al. The 3D sagittal profile of thoracic versus lumbar major curves in adolescent idiopathic scoliosis. Spine Deform. 2019;7:60-5.

22. Bernhardt M, Bridwell K. Segmental analysis of the sagittal plane alignment of the normal thoracic and lumbar spines and thoracolumbar junction. Spine. 1989;14:717-21.

23. Sanders J. Normal growth of the spine and skeletal maturation. Semin Spine Surg. 2015;27:16-20.

24. Gardner A, Archer J, Berryman F, Pynsent P. The resting coronal and sagittal stance position of the torso in adolescents with and without spinal deformity. Sci Rep. 2021;11:2354.

25. Poussa M, Heliövaara M, Seitsamo J, et al. Development of spinal posture in a cohort of children from the age of 11 to 22 years. Eur Spine J. 2005;14:738-42.

26. De La Rocha A, McClung A, Sucato D. Increased body mass index negatively affects patient satisfaction after a posterior fusion and instrumentation for adolescent idiopathic scoliosis. Spine Deform. 2014;2:208-13.

27. Walicka-Cupryś K, Skalska-Izdebska R, Rachwal M, Truszczńska A. Influence of the weight of a school backpack on spinal curvature in the sagittal plane of seven-year-old children. Biomed Res Int. 2015; 2015: 817913.

\section{Figures}




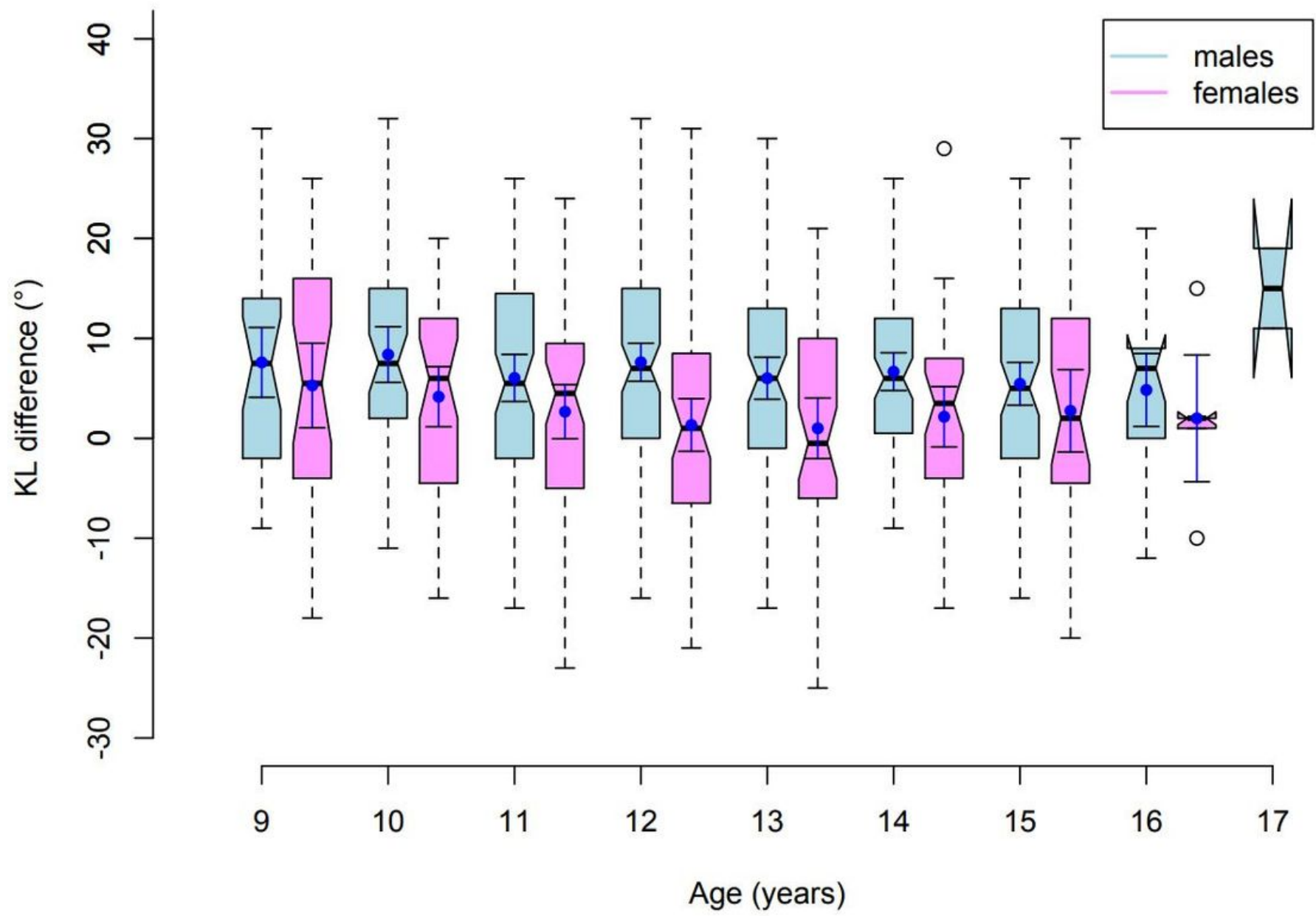

Figure 1

The $\mathrm{KL}$ difference $\left(^{\circ}\right)$ for males and females versus age (years). The thick horizontal line at the pinch point represents the median value with the box surrounding that the interquartile range. The solid dark blue dot and associated bars represent the mean and 95\% confidence interval for the mean. The dotted line and whiskers are up to 1.5 times the interquartile range and any data beyond that is seen as an open circle. 


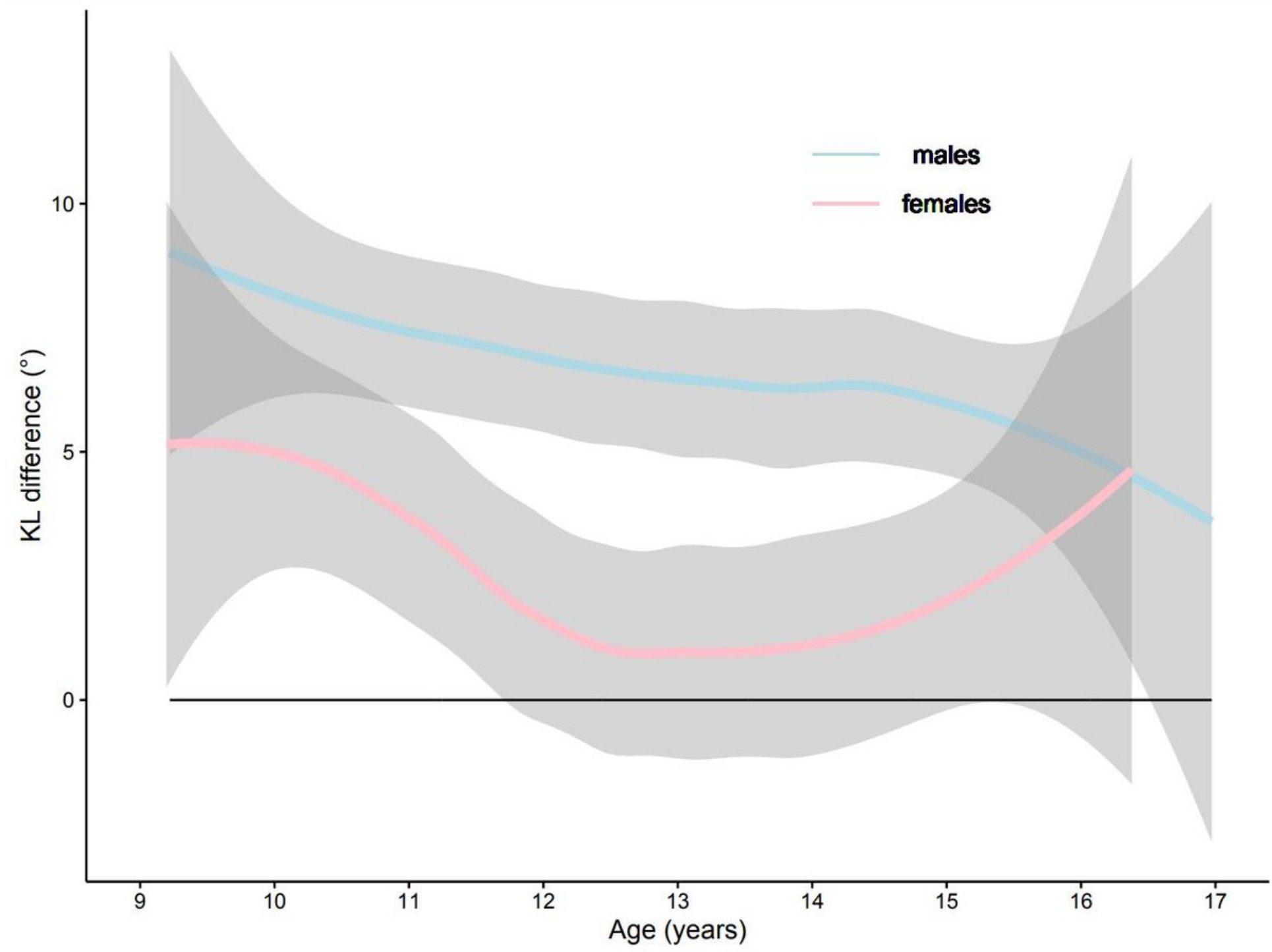

Figure 2

The $\mathrm{KL}$ difference loess lines only $\left({ }^{\circ}\right)$ with $95 \%$ confidence intervals for males and females versus age (years). 